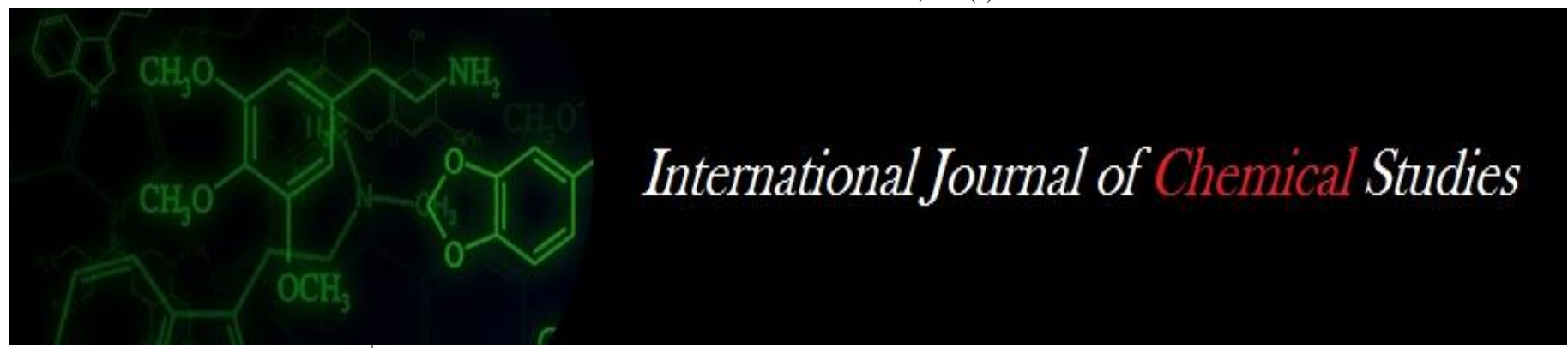

P-ISSN: 2349-8528

E-ISSN: 2321-4902

www.chemijournal.com

IJCS 2020; SP-8(3): 05-08

(C) 2020 IJCS

Received: 04-04-2020

Accepted: 06-05-2020

\section{RN Nishad}

Department of Entomology,

ANDUA\&T, Kumarganj,

Ayodhya, Uttar Pradesh, India

RB Singh

Department of Entomology,

ANDUA\&T, Kumarganj,

Ayodhya, Uttar Pradesh, India

Suraj Kumar

Department of Entomology,

ANDUA\&T, Kumarganj,

Ayodhya, Uttar Pradesh, India

Sachin Kumar Yadav

Department of Entomology,

ANDUA\&T, Kumarganj,

Ayodhya, Uttar Pradesh, India
Corresponding Author:

RN Nishad

Department of Entomology,

ANDUA\&T, Kumarganj,

Ayodhya, Uttar Pradesh, India

\section{Eco-friendly management of pulse beetle, Callosobruchus chinensis Linn. of stored chickpea seed}

\author{
RN Nishad, RB Singh, Suraj Kumar and Sachin Kumar Yadav
}

DOI: https://doi.org/10.22271/chemi.2020.v8.i3a.9751

\begin{abstract}
The experiment was conducted under laboratory condition in the Department of Entomology, Acharya Narendra Deva University of Agricultural and technology, Kumarganj, Ayodhya to find out the ecofriendly management of pulse beetle, $C$. chinensis Linn. in stored chickpea $c v$; PG-186 seed under ambient condition by using some promising botanicals as seed protectant viz., neem oil @ $5 \mathrm{ml} / \mathrm{kg}$ seed, mustard oil@ @ ml $/ \mathrm{kg}$ seed, coconut oil @ $5 \mathrm{ml} / \mathrm{kg}$ seed, citronella oil @ $5 \mathrm{ml} / \mathrm{kg}$ seed, karanj leaf powder $5 \mathrm{~g} / \mathrm{kg}$ seed, marigold leaf powder $5 \mathrm{~g} / \mathrm{kg}$ seed, lemon grass leaf powder $5 \mathrm{~g} / \mathrm{kg}$ seed, neem leaf powder $5 \mathrm{~g} / \mathrm{kg}$ seed and deltamethrin $2.8 \mathrm{EC}$ as check @ $0.04 \mathrm{ml} / \mathrm{kg}$ seed. Among tested all seed protectants besides chemicals, deltamethrin 2.8 EC; Neem oil @ $5 \mathrm{ml} \mathrm{kg}$-1seed followed by Mustaard oil @ $5 \mathrm{ml} \mathrm{kg-1} \mathrm{seed} \mathrm{was} \mathrm{found} \mathrm{most} \mathrm{effective} \mathrm{botanical} \mathrm{as} \mathrm{seed} \mathrm{protectant} \mathrm{in} \mathrm{due} \mathrm{to} \mathrm{less} \mathrm{seed} \mathrm{damage} \mathrm{and}$ weight loss with high germination percent i.e. above IMSCS upto 9 month of chickpea storage periods.
\end{abstract}

Keywords: Callosobruchus chinensis, chickpea (Cicer arietinum), botanical seed protectants, and ecofriendly management

\section{Introduction}

Pulses, the "wonderful gift of nature" play an important role in Indian economy and are a rich source of supplementary protein of daily diets of vegetarian population. The pulses have played a vital role in the improvement of agricultural economy of different countries (Sarwar et al. 2003 and Deeba et al. 2006) ${ }^{[16,6]}$. Chickpea (Cicer arietinum L.) is an ancient crop and used as a good source of energy i.e. 416 calories/100 gm chickpea (Shrestha, 2001) ${ }^{[17]}$ along with protein $(18-22 \%)$, carbohydrate $(52-70 \%)$, fat (4-10\%), minerals (calcium, phosphorus, iron) and vitamins (Ali and Prasad, 2002) ${ }^{[1]}$. In India chickpea grow on about $10.56 \mathrm{~m}$ ha with production of $11.23 \mathrm{~m}$ tonnes and productivity level of $1063 \mathrm{~kg} / \mathrm{ha}$ in $2017-18$ respectively (Anonymous, 2018-19) ${ }^{[2]}$. Besides production constraints, post-harvest loss of chickpea is very high in farmers' storage conditions. The major factor of the heavy loss of the grain legumes in the storage is pulse beetle (C. chinensis L.) (Southgate, 1978; Talekar, 1988) ${ }^{[19,20]}$. Botanicals seed protectants are known to suppress the feeding and breeding of insects in various ways in addition to causing direct mortality. Some farmers use seed protectants such as various type of neem based product mustard oil and various species of plants, a few of which have been found to provide adequate protection for longer duration against pulse beetle $(C$. chinensis) insect (Golob and Webley, 1980) ${ }^{[7]}$. Among the other protection techniques the use of plant part powders edible oils and plant powder as grain protectant is an age old practice and gaining rapid popularity providing safer conditions to human health, easy to handle and with no deleterious effect on stored products.

Therefore, the present studies have been proposed to explore the possibility of using some plant material as seed protectants against $C$. chinensis.

\section{Material and Methods}

The experiment was conducted in CRD with 10 treatments including control and three replications during the year 2018-19 and 2019-20. For this experiment $30 \mathrm{~kg}$ disinfested seed of chickpea variety PG-186 was used. Five hundred gram of chickpea seed was taken for each replication in each treatment. 
Required quantities of seed protectant was mix with water @ $5 \mathrm{ml} / \mathrm{kg}$ seed for proper seed coating. After mixing the protectants, seed packed in $1 \mathrm{~kg}$ capacity of Jute bags and mouth of bags were tight with the help of thread (Sutali). The bags were kept on racks under ambient conditions on racks in the laboratory of seed entomology. The observations were recorded on 3, 6, and 9 months of storage.

\section{Percent Seed Moisture content}

Seed moisture content of chickpea in each replication of each seed protectants was recorded with the help of MAC Electronic Moisture Meter.

Whereas, Seed damage, Seed germination and percentage weight loss of seed were worked out as bellow:

\section{Percent Seed damage}

For damage, hundred seed from each replication of each treatment were carefully examined for observing damaged seed by the bruchid pests with the help of magnifying lens (10 $\mathrm{X})$. The obtained data were computed to work out per cent damaged seed by $C$. chinensis from following formula (Kumar, 2008) ${ }^{[10]}$.

Percent seed damage $=\frac{\text { Number of holed seed in sample }}{\text { Total No. of seed in sample }} \times 100$

\section{Percent Seed germination}

Germination paper (Towel paper) method (ISTA, 1976) was used to know the germination of chickpea seed and calculate the percent seed germination with help of following formula.

Per cent seed germination $=\frac{\text { Number of germinated seed in sample }}{\text { Total number of seed in sample for germination }} \times 100$

\section{Percent weight loss of seed}

To calculate the per cent weight loss of seed, 100 were taken from each replication of each treatment and carefully examined with the help of magnifying lens (10x) to separate out the bored seed. The observations were recorded at the end of experiment (180 days). The data thus obtained will be used for computing weight loss per cent by using above formula (Dawae, 2008) ${ }^{[5]}$

Per cent weight loss in seed $=\frac{\text { Weight of damage seed of sample }}{\text { Total weight of seed in sample }} \times 100$

\section{Result and Discussion}

\section{Percent seed moisture content}

As perusal of results (Table- 1, $2 \& 3$ ) reveals that the percent seed moisture content was ranged from 11.8 to 14.48 percent. The level of seed moisture content was directly related to environmental conditions and storage period.
The maximum seed moisture content percent were observed in Karanj leaf powder (14.09) and Marigold leaf powder (14.08), both are statistically at par with each other followed by Neem leaf powder (13.94). The minimum percent moisture was recorded in Deltamethrin 2.8 EC @ $0.04 \mathrm{ml} \mathrm{kg}^{-1}$ (12.81\%) followed by Mustard oil (13.32\%) and Citronella oil $(13.51 \%)$. More or less similar work has also been reported by Nishad et al. (2017) ${ }^{[13,14]}$, Pal and Katiyar (2013) ${ }^{[12]}$. The significant difference of seed moisture content per cent was observed among seed protectants.

\section{Percent insect seed damage and weight loss}

The result (Table- 1, 2 \& 3) showed that a considerable variation was observed in insect damage in different protectants up to 9 months stored chickpea seed. All treatment was significantly superior then the untreated control that was maximum insect damage $(14.5 \%)$ at 9 month of storage period.

The percent insect damaged ranged 0.50 to 9.17 percent. The maximum seed damage was recorded in Marigold leaf powder @ $5 \mathrm{~g} \mathrm{~kg}^{-1}$ seed with 9.17 percent followed by Lemon grass leaf powder @ $5 \mathrm{~g} \mathrm{~kg}^{-1}$ seed with 8.50 percent and Neem leaf powder@5 $\mathrm{g} \mathrm{kg}^{-1}$ with 7.16 percent. The minimum seed damage was recorded in Deltamethrin 2.8 EC @ $0.04 \mathrm{ml} \mathrm{kg}^{-1}$ seed with 2.83 percent followed by Neem oil @ $5 \mathrm{ml} \mathrm{kg}^{-1}$ seed with 4.33 percent and Mustard oil @ $5 \mathrm{ml} \mathrm{kg}^{-1}$ seed with 4.7 percent

Singh et al. (2017) ${ }^{[13]}$ and Bajiya (2010) ${ }^{[4]}$ were also found significant reduction in grains damage as well as loss in weight by $C$. chinensis with neem kernel powder. The current research of plant based insecticides against $C$. chinensis in pulse storage are also supported by Khinchi et al., (2017) ${ }^{[9]}$, Khan et al., (2015) ${ }^{[8]}$, Rugumamu (2015) ${ }^{[15]}$ and Tesema et al., (2015) ${ }^{[21]}$.

\section{Percent seed germination}

The results (Table- 1, $2 \& 3$ ) showed significant differences in percent seed germination of different protectants over a span of 3, 6 and 9 months of storage. The germination percentage was decreased in all treatments with increased in storage period of chickpea seed.

The maximum seed germination percent were recorded in Deltamethrin 2.8 EC @ $0.04 \mathrm{ml} \mathrm{kg}^{-1}$ with 83.83 percent and Neem oil @ $5 \mathrm{ml} \mathrm{kg}^{-1}$ with 82.83 percent which were statistically at par with each other followed by Mustard oil @ $5 \mathrm{ml} \mathrm{kg}^{-1}$ with 82.50 percent and Citronella oil @ $5 \mathrm{ml} \mathrm{kg}^{-1}$ with 81.16 percent. The minimum germination percent were observed in Marigold leaf powder @ $5 \mathrm{~g} \mathrm{~kg}^{-1}$ with 75.33 percent followed by Lemon grass leaf powder @ $5 \mathrm{~g} \mathrm{~kg}^{-1}$ with 76.50 percent. These results were also supported by Lal and Raj (2012) ${ }^{[11]}$ and Singh et al. (2014) ${ }^{[18]}$ in Pigeonpea, and Nishad et al. (2017) ${ }^{[13,14]}$ in chickpea seed.

Table 1: Effect of various eco-friendly seed protectants against $C$. chinensis under ambient storage condition after 3 months of ambient storage during the year 2018-19 and 2019-20.

\begin{tabular}{|c|c|c|c|c|c|c|c|c|c|c|c|c|c|}
\hline \multirow{3}{*}{ S.N. } & \multirow{3}{*}{ Name of the Treatments } & \multicolumn{12}{|c|}{$\begin{array}{c}\text { Average percent seed moisture content , insect damage, seed germination and seed Weight loss } \\
\text { after } 3 \text { months of ambient storage condition }\end{array}$} \\
\hline & & \multicolumn{3}{|c|}{ Seed Moisture Content } & \multicolumn{3}{|c|}{ Damage seed } & \multicolumn{3}{|c|}{ Seed Germination } & \multicolumn{3}{|c|}{ Seed Weight loss } \\
\hline & & 2018-19 & 2019-20 & $* \mathbf{P . M}$. & 2018-19 & 2019-20 & P.M. & 2018-19 & 2019-20 & P.M. & 2018-19 & 2019-20 & P.M. \\
\hline 1 & Neem oil & $\begin{array}{c}11.2 \\
(19.53)\end{array}$ & $\begin{array}{c}11.1 \\
(19.45)\end{array}$ & 11.15 & $\begin{array}{c}1.00 \\
(5.73)\end{array}$ & $\begin{array}{l}1.33 \\
(6.53)\end{array}$ & 1.16 & $\begin{array}{c}91.33 \\
(72.85)\end{array}$ & $\begin{array}{c}90.67 \\
(72.18)\end{array}$ & 91.00 & $\begin{array}{c}3.67 \\
(11.08)\end{array}$ & $\begin{array}{c}4.21 \\
(11.81)\end{array}$ & 3.94 \\
\hline 2 & C & $\begin{array}{c}11.7 \\
(19.99)\end{array}$ & $\begin{array}{c}11.6 \\
(19.89)\end{array}$ & 11.65 & $\begin{array}{l}2.00 \\
(8.12)\end{array}$ & $\begin{array}{l}2.33 \\
(8.74)\end{array}$ & 2.16 & $\begin{array}{c}88.00 \\
(69.71)\end{array}$ & $\begin{array}{c}88.33 \\
(70.02)\end{array}$ & 88.16 & $\begin{array}{c}5.33 \\
(13.29)\end{array}$ & $\begin{array}{c}5.73 \\
(13.79)\end{array}$ & 5.53 \\
\hline 3 & Mustard oil & $\begin{array}{c}11.4 \\
(19.72)\end{array}$ & $\begin{array}{c}11.3 \\
(19.62)\end{array}$ & 11.35 & $\begin{array}{c}1.33 \\
(6.53)\end{array}$ & $\begin{array}{c}1.67 \\
(7.33)\end{array}$ & 1.50 & $\begin{array}{c}90.67 \\
(72.21)\end{array}$ & $\begin{array}{c}89.33 \\
(70.94)\end{array}$ & 90.00 & $\begin{array}{c}4.52 \\
(12.24)\end{array}$ & $\begin{array}{c}4.95 \\
(12.87)\end{array}$ & 4.73 \\
\hline 4 & Citronella oil & 11.6 & 11.5 & 11.55 & 1.67 & 2.00 & 1.83 & 89.33 & 88.67 & 89.00 & 4.83 & 5.34 & 5.08 \\
\hline
\end{tabular}




\begin{tabular}{|c|c|c|c|c|c|c|c|c|c|c|c|c|c|}
\hline & & $(19.89)$ & $(19.81)$ & & $(7.33)$ & $(8.12)$ & & $(70.94)$ & $(70.32)$ & & $(12.65)$ & $(13.30)$ & \\
\hline 5 & Karanj leaf powder & $\begin{array}{c}12.1 \\
(20.34)\end{array}$ & $\begin{array}{c}12.0 \\
(20.25)\end{array}$ & 12.05 & $\begin{array}{c}2.33 \\
(8.74) \\
\end{array}$ & $\begin{array}{c}2.67 \\
(9.35)\end{array}$ & 2.50 & $\begin{array}{c}87.67 \\
(69.42) \\
\end{array}$ & $\begin{array}{c}87.33 \\
(69.14)\end{array}$ & 87.00 & $\begin{array}{c}6.39 \\
(14.64)\end{array}$ & $\begin{array}{c}6.87 \\
(15.17)\end{array}$ & 6.63 \\
\hline 6 & Marigold leaf powder & $\begin{array}{c}12.6 \\
(20.77) \\
\end{array}$ & $\begin{array}{c}12.7 \\
(20.86)\end{array}$ & 12.65 & $\begin{array}{c}3.67 \\
(11.01) \\
\end{array}$ & $\begin{array}{c}4.00 \\
(11.53) \\
\end{array}$ & 3.83 & $\begin{array}{c}85.67 \\
(67.74) \\
\end{array}$ & $\begin{array}{c}85.33 \\
(67.74) \\
\end{array}$ & 85.50 & $\begin{array}{c}8.86 \\
(17.27) \\
\end{array}$ & $\begin{array}{c}9.21 \\
(17.64) \\
\end{array}$ & 9.03 \\
\hline 7 & Lemongrass leaf powder & $\begin{array}{c}12.6 \\
(20.78) \\
\end{array}$ & $\begin{array}{c}12.5 \\
(20.69)\end{array}$ & 12.55 & $\begin{array}{c}3.00 \\
(9.79) \\
\end{array}$ & $\begin{array}{c}3.67 \\
(10.95) \\
\end{array}$ & 3.33 & $\begin{array}{c}86.33 \\
(68.29) \\
\end{array}$ & $\begin{array}{c}86.67 \\
(68.57) \\
\end{array}$ & 86.50 & $\begin{array}{c}7.67 \\
(16.10)\end{array}$ & $\begin{array}{c}8.36 \\
(16.80)\end{array}$ & 8.01 \\
\hline 8 & Neem leaf powder & $\begin{array}{c}12.4 \\
(20.60)\end{array}$ & $\begin{array}{c}12.3 \\
(20.51)\end{array}$ & 12.35 & $\begin{array}{c}2.67 \\
(9.35)\end{array}$ & $\begin{array}{c}3.33 \\
(10.49)\end{array}$ & 3.00 & $\begin{array}{c}86.67 \\
(68.56)\end{array}$ & $\begin{array}{c}85.33 \\
(67.46)\end{array}$ & 86.00 & $\begin{array}{c}6.95 \\
(15.25)\end{array}$ & $\begin{array}{c}7.67 \\
(16.06)\end{array}$ & 7.31 \\
\hline 9 & Deltamethrin (2.8 EC) Check & $\begin{array}{c}10.8 \\
(19.17)\end{array}$ & $\begin{array}{c}10.9 \\
(19.26)\end{array}$ & 10.85 & $\begin{array}{c}0.33 \\
(1.91)\end{array}$ & $\begin{array}{c}0.67 \\
(3.82)\end{array}$ & 0.50 & $\begin{array}{c}92.33 \\
(73.92)\end{array}$ & $\begin{array}{c}91.67 \\
(73.29)\end{array}$ & 92.00 & $\begin{array}{c}2.36 \\
(8.84) \\
\end{array}$ & $\begin{array}{c}2.89 \\
(9.74)\end{array}$ & 2.62 \\
\hline 10 & Control & $\begin{array}{c}12.9 \\
(21.03)\end{array}$ & $\begin{array}{c}12.8 \\
(20.94)\end{array}$ & 12.85 & $\begin{array}{c}4.67 \\
(12.45) \\
\end{array}$ & $\begin{array}{c}5.00 \\
(12.91) \\
\end{array}$ & 4.83 & $\begin{array}{c}84.33 \\
(66.66) \\
\end{array}$ & $\begin{array}{c}83.67 \\
(66.15)\end{array}$ & 84.00 & $\begin{array}{c}10.67 \\
(19.04)\end{array}$ & $\begin{array}{c}11.23 \\
(19.55)\end{array}$ & 10.95 \\
\hline & CD 5\% & 1.16 & 1.13 & 1.14 & 2.33 & 2.47 & 2.40 & 1.94 & 2.15 & 2.04 & 0.95 & 0.97 & 0.96 \\
\hline & SEm \pm & 0.39 & 0.38 & 0.38 & 0.78 & 0.83 & 0.80 & 0.65 & 0.72 & 0.68 & 0.32 & 0.33 & 0.32 \\
\hline
\end{tabular}

The figures given in parenthesis angular transformed value.

*P.M. = Pooled Mean

Table 2: Effect of various eco-friendly seed protectants against $C$. chinensis under ambient storage condition after 6 months of ambient storage during the year 2018-19 and 2019-20.

\begin{tabular}{|c|c|c|c|c|c|c|c|c|c|c|c|c|c|}
\hline \multirow{3}{*}{ S.N. } & \multirow{3}{*}{ Name of the Treatments } & \multicolumn{12}{|c|}{$\begin{array}{l}\text { Average percent seed moisture content, insect damage, seed germination and seed Weight loss } \\
\text { after } 6 \text { months of ambient storage condition }\end{array}$} \\
\hline & & \multicolumn{3}{|c|}{ Seed Moisture Content } & \multicolumn{3}{|c|}{ Damage seed } & \multicolumn{3}{|c|}{ Seed Germination } & \multicolumn{3}{|c|}{ Seed Weight loss } \\
\hline & & 2018-19 & 2019-20 & P.M. & 2018-19 & 2019-20 & P.M. & 2018-19 & 2019-20 & P.M. & 2018-19 & 2019-20 & P.M. \\
\hline 1 & Neem oil & $\begin{array}{c}12.2 \\
(20.43) \\
\end{array}$ & $\begin{array}{c}12.1 \\
(20.34) \\
\end{array}$ & 12.15 & $\begin{array}{c}2.33 \\
(8.74)\end{array}$ & $\begin{array}{c}2.67 \\
(9.35)\end{array}$ & 2.50 & & & 88.00 & $\begin{array}{c}6.67 \\
(14.95)\end{array}$ & $\begin{array}{c}8.34 \\
(16.76)\end{array}$ & 7.50 \\
\hline 2 & Coco & $\begin{array}{c}12.7 \\
(20.86)\end{array}$ & & 12.65 & & & 4.00 & & & 85.83 & & & 10.94 \\
\hline 3 & Must & $\begin{array}{c}12.5 \\
(20.69) \\
\end{array}$ & $\begin{array}{c}12.4 \\
(20.60) \\
\end{array}$ & 12.45 & $\begin{array}{c}2.67 \\
(9.35)\end{array}$ & & 2.83 & & & 87.67 & $\begin{array}{c}8.33 \\
(16.72) \\
\end{array}$ & & 8.97 \\
\hline 4 & 0 & $\begin{array}{c}12.8 \\
(20.94) \\
\end{array}$ & $\begin{aligned} 12 \\
(20 .\end{aligned}$ & 2.80 & $\begin{array}{r}3 . \\
(10\end{array}$ & $\begin{array}{r}3 . \\
(11 \\
\end{array}$ & 3.50 & $\begin{array}{r}86 \\
(68 \\
\end{array}$ & & 86.16 & $\begin{array}{l}6 \\
11) \\
\end{array}$ & $\begin{array}{l}8 \\
23) \\
\end{array}$ & 9.37 \\
\hline 5 & Kar & $\begin{array}{c}13.2 \\
(21.29) \\
\end{array}$ & $\begin{array}{c}13.1 \\
(21.20) \\
\end{array}$ & 13.15 & $\begin{array}{c}4.00 \\
(11.47) \\
\end{array}$ & $\begin{array}{c}4.67 \\
(12.45) \\
\end{array}$ & 4.33 & $\begin{array}{r}85 \\
(67 \\
\end{array}$ & $\begin{array}{r}84 \\
(66 \\
\end{array}$ & 84.83 & $\begin{array}{l}6 \\
2) \\
\end{array}$ & $\begin{array}{l}3 \\
8) \\
\end{array}$ & 13.04 \\
\hline 6 & Mari & $\begin{array}{c}13.6 \\
(21.63)\end{array}$ & $\begin{array}{c}13.7 \\
(21.70)\end{array}$ & 13.65 & $\begin{array}{c}5.00 \\
(12.87)\end{array}$ & $\begin{array}{c}6.67 \\
(14.94)\end{array}$ & 5.83 & & & 81.33 & & & 14.01 \\
\hline 7 & Lemongrass & $\begin{array}{c}13.5 \\
(21.54) \\
\end{array}$ & $\begin{array}{r}1 \\
(21 \\
\end{array}$ & 1. & $\begin{array}{r}4 . \\
(12 \\
\end{array}$ & $\begin{array}{r}5 . \\
(13 \\
\end{array}$ & 5.00 & $\begin{array}{r}8 \\
(6 \\
\end{array}$ & $\begin{array}{r}8 \\
(6 \\
\end{array}$ & 82.00 & & & 14.90 \\
\hline 8 & er & $\begin{array}{c}13.3 \\
(21.37) \\
\end{array}$ & $\begin{array}{c}13.4 \\
(21.45) \\
\end{array}$ & 13.35 & $\begin{array}{c}4.33 \\
(11.99) \\
\end{array}$ & $\begin{array}{c}5.00 \\
(12.87) \\
\end{array}$ & 4.66 & $\begin{array}{c}83.67 \\
(66.15) \\
\end{array}$ & $\begin{array}{c}82.33 \\
(65.15) \\
\end{array}$ & 83.00 & $\begin{array}{r}13 \\
(21 \\
\end{array}$ & $\begin{array}{r}14 \\
(22 \\
\end{array}$ & 13.82 \\
\hline 9 & Deltamet & $\begin{array}{c}11.3 \\
(19.63) \\
\end{array}$ & $\begin{array}{c}11.2 \\
(19.53)\end{array}$ & 11.25 & $\begin{array}{c}1.67 \\
(7.33)\end{array}$ & $\begin{array}{c}1.33 \\
(6.53) \\
\end{array}$ & 1.50 & $\begin{array}{c}89.33 \\
(70.23) \\
\end{array}$ & $\begin{array}{c}88.33 \\
(69.71) \\
\end{array}$ & 88.83 & $\begin{array}{c}5.67 \\
(13.84)\end{array}$ & $\begin{array}{c}6.31 \\
(14.56)\end{array}$ & 5.99 \\
\hline 10 & (1) & $\begin{array}{c}13.9 \\
(21.87) \\
\end{array}$ & $\begin{array}{c}14.1 \\
(22.04) \\
\end{array}$ & 14.0 & $\begin{array}{c}9.33 \\
(17.77) \\
\end{array}$ & $\begin{array}{c}8.67 \\
(17.10) \\
\end{array}$ & 9.00 & $\begin{array}{c}79.67 \\
(63.19) \\
\end{array}$ & $\begin{array}{c}79.33 \\
(62.93) \\
\end{array}$ & 79.50 & $\begin{array}{c}19.34 \\
(26.05) \\
\end{array}$ & $\begin{array}{c}20.45 \\
(26.86) \\
\end{array}$ & 19.89 \\
\hline & CD $5 \%$ & 0.91 & & (1.00 & 1.82 & 1.80 & 1.81 & 1.99 & & 1.84 & 1.33 & & 1.36 \\
\hline & SEm \pm & 0.38 & 0.40 & 0.39 & 0.61 & 0.60 & 0.61 & 0.67 & 0.57 & 0.62 & 0.45 & 0.47 & 0.46 \\
\hline
\end{tabular}

The figures given in parenthesis angular transformed value.

*P.M. = Pooled Mean

Table 3: Effect of various eco-friendly seed protectants against $C$. chinensis under ambient storage condition after 9 months of ambient storage during the year 2018-19 and 2019-20.

\begin{tabular}{|c|c|c|c|c|c|c|c|c|c|c|c|c|c|}
\hline \multirow{3}{*}{ S.N. } & \multirow{3}{*}{ Name of the Treatments } & \multicolumn{12}{|c|}{$\begin{array}{l}\text { Average percent seed moisture content, insect damage, seed germination and seed Weight loss } \\
\text { after } 9 \text { months of ambient storage condition }\end{array}$} \\
\hline & & \multicolumn{3}{|c|}{ Seed Moisture Content } & \multicolumn{3}{|c|}{ Damage seed } & \multicolumn{3}{|c|}{ Seed Germination } & \multicolumn{3}{|c|}{ Seed Weight loss } \\
\hline & & 2018-19 & 2019-20 & P.M. & 2018-19 & 2019-20 & P.M. & 2018-19 & 2019-20 & P.M. & 2018-19 & 2019-20 & P.M. \\
\hline 1 & Neem oil & $\begin{array}{c}13.1 \\
(2120)\end{array}$ & $\begin{array}{c}13.2 \\
(21.29)\end{array}$ & 13.15 & $\begin{array}{c}4.33 \\
(11.99)\end{array}$ & $\begin{array}{c}4.33 \\
(11.99)\end{array}$ & 4.33 & $\begin{array}{c}83.33 \\
(65.88)\end{array}$ & $\begin{array}{c}82.33 \\
(65.12)\end{array}$ & 82.83 & $\begin{array}{c}14.32 \\
(22.20)\end{array}$ & \begin{tabular}{c|c}
14.45 \\
$(22.34)$
\end{tabular} & 14.38 \\
\hline 2 & Coconut oil & $\begin{array}{c}13.6 \\
(21.63)\end{array}$ & $\begin{array}{c}13.7 \\
(21.70)\end{array}$ & 13.65 & $\begin{array}{c}6.00 \\
(14.14)\end{array}$ & $\begin{array}{c}6.33 \\
(14.56)\end{array}$ & 6.16 & & & 81.16 & $\begin{array}{l}17.46 \\
(24.65)\end{array}$ & & 17.99 \\
\hline 3 & Mustard oil & $\begin{array}{c}13.3 \\
(21.37) \\
\end{array}$ & $\begin{array}{c}13.4 \\
(21.45) \\
\end{array}$ & 13.35 & $\begin{array}{c}4.67 \\
(12.45) \\
\end{array}$ & $\begin{array}{c}4.67 \\
(12.45) \\
\end{array}$ & 4.67 & $\begin{array}{c}82.33 \\
(65.38) \\
\end{array}$ & & 82.50 & $\begin{array}{c}15.96 \\
(23.53) \\
\end{array}$ & \begin{tabular}{|c|}
15.78 \\
$(23.40)$ \\
\end{tabular} & 15.87 \\
\hline 4 & Citro & $\begin{array}{c}13.5 \\
(21.54)\end{array}$ & $\begin{array}{c}13.6 \\
(21.63)\end{array}$ & 13.55 & $\begin{array}{c}5.33 \\
(13.33)\end{array}$ & $\begin{array}{c}5.67 \\
(13.75)\end{array}$ & 5.50 & $\begin{array}{c}82.00 \\
(64.87)\end{array}$ & $\begin{array}{c}80.33 \\
(63.65)\end{array}$ & 81.16 & $\begin{array}{c}16.37 \\
(23.85)\end{array}$ & $\begin{array}{c}16.82 \\
(24.21)\end{array}$ & 16.59 \\
\hline 5 & Karanj leaf powder & $\begin{array}{c}14.2 \\
(22.12) \\
\end{array}$ & $\begin{array}{c}14.3 \\
(22.20) \\
\end{array}$ & 14.25 & $\begin{array}{c}6.67 \\
(14.94) \\
\end{array}$ & $\begin{array}{c}6.33 \\
(14.56) \\
\end{array}$ & 6.50 & $\begin{array}{c}81.67 \\
(64.62) \\
\end{array}$ & $\begin{array}{c}80.67 \\
(63.89) \\
\end{array}$ & 81.17 & $\begin{array}{c}19.21 \\
(25.98) \\
\end{array}$ & $\begin{array}{c}20.16 \\
(26.65) \\
\end{array}$ & 20.58 \\
\hline 6 & Marigold leaf $\mathrm{p}$ & $\begin{array}{c}14.1 \\
(22.04)\end{array}$ & $\begin{array}{c}14.1 \\
(22.04)\end{array}$ & 14.10 & $\begin{array}{c}8.67 \\
(17.09)\end{array}$ & $\begin{array}{c}9.67 \\
(18.10)\end{array}$ & 9.17 & $\begin{array}{c}76.33 \\
(60.87)\end{array}$ & $\begin{array}{c}74.33 \\
(59.54)\end{array}$ & 75.33 & $\begin{array}{c}21.67 \\
(27.71)\end{array}$ & $\begin{array}{c}21.96 \\
(27.92)\end{array}$ & 21.81 \\
\hline 7 & Lemongrass leaf powder & $\begin{array}{c}13.9 \\
(21.98)\end{array}$ & $\begin{array}{c}13.9 \\
(21.98)\end{array}$ & 13.90 & $\begin{array}{c}8.33 \\
(16.40)\end{array}$ & $\begin{array}{c}8.67 \\
(17.10) \\
\end{array}$ & 8.50 & $\begin{array}{c}77.33 \\
(61.54) \\
\end{array}$ & $\begin{array}{c}75.67 \\
(60.42) \\
\end{array}$ & 76.50 & $\begin{array}{c}22.56 \\
(28.34) \\
\end{array}$ & $\begin{array}{c}23.17 \\
(28.75) \\
\end{array}$ & 22.86 \\
\hline 8 & Neem leaf powder & 13.8 & 13.7 & 13.75 & 7.33 & 7.00 & 7.16 & 78.67 & 77.67 & 78.17 & 20.47 & 21.26 & 20.86 \\
\hline
\end{tabular}




\begin{tabular}{|c|c|c|c|c|c|c|c|c|c|c|c|c|c|}
\hline & & $(21.87)$ & $(21.71)$ & & $(15.67)$ & $(16.06)$ & & $(62.47)$ & $(61.77)$ & & $(26.88)$ & $(27.44)$ & \\
\hline 9 & Deltamethrin (2.8 EC) & $\begin{array}{c}12.8 \\
(20.94)\end{array}$ & $\begin{array}{c}12.5 \\
(22.69)\end{array}$ & 12.65 & $\begin{array}{c}2.67 \\
(9.35)\end{array}$ & $\begin{array}{c}3.00 \\
(9.87)\end{array}$ & 2.83 & $\begin{array}{c}84.67 \\
(66.93)\end{array}$ & $\begin{array}{c}83.00 \\
(65.62)\end{array}$ & 83.83 & $\begin{array}{c}11.33 \\
(19.75)\end{array}$ & $\begin{array}{c}12.67 \\
(20.89)\end{array}$ & 12.00 \\
\hline 10 & Con & $\begin{array}{c}15.1 \\
(22.85) \\
\end{array}$ & $\begin{array}{c}14.9 \\
(22.69) \\
\end{array}$ & 15.00 & $\begin{array}{c}14.67 \\
(22.50) \\
\end{array}$ & $\begin{array}{c}14.33 \\
(22.23) \\
\end{array}$ & 14.50 & $\begin{array}{c}71.33 \\
(57.61) \\
\end{array}$ & $\begin{array}{c}70.67 \\
(57.18) \\
\end{array}$ & 71.00 & $\begin{array}{c}33.65 \\
(35.41) \\
\end{array}$ & $\begin{array}{c}34.56 \\
(35.99) \\
\end{array}$ & 34.10 \\
\hline & CD 5\% & 0.94 & 0.93 & 0.93 & 1.64 & 1.42 & 1.53 & 1.44 & 1.51 & 1.47 & 1.85 & 1.89 & 1.87 \\
\hline & SEm \pm & 0.31 & 0.31 & 0.31 & 0.55 & 0.79 & 0.67 & 0.48 & 0.50 & 0.49 & 0.62 & 0.63 & 0.62 \\
\hline
\end{tabular}

The figures given in parenthesis angular transformed value.

*P.M. = Pooled Mean

\section{Conclusion}

During evaluation all tested botanical seed protectants were capable to maintained the seed germination above IMSCS nine months of ambient storage of chickpea which were found significantly superior over control in reducing the seed infestation and weight loss. Besides, chemical seed protctant

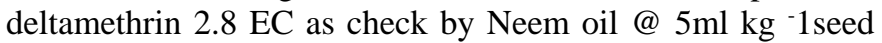
with 82.83 percent and Mustard oil @ $5 \mathrm{ml} \mathrm{kg}^{-1}$ with 82.50 percent maintained maximum seed germination with comparatively minimum bruchids infestation and weight loss upto nine month of storage.

Thus the present investigation advocated that Neem oil @ $5 \mathrm{ml} / \mathrm{kg}$ seed and Mustad oil @ 5ml/ kg seed may be utilizes as suitable eco-friendly seed protectants in stored chickpea against pulse beetle, Callosobruchus chinensis Linn. For a long period of 9 months.

\section{Reference}

1. Ali SI, Prasad R. Rabi pulses: chickpea, lentil, Lathyrus and French bean. In: R. Prasad (ed.), Textbook of Field Crops Production. New Delhi: Directorate of Information and Publication of Agriculture, Indian Council of Agriculture Research, 2002, 317-371.

2. Anonymous, Agricultural Statistics at a Glance. Department of Agriculture and Cooperation, Ministry of Agriculture, Government of India, 2018-19.

3. Anonymous. Germination method part I agriculture and horticulture seeds. Seed Science and Technology. 1976; 4:120.

4. Bajiya RS. Bio-ecology and Management of Pulse Beetle, Callosobruchus chinensis (Linn.) on Mungbean, Vigna radiata (Linn.) Wilczek Ph.D. Thesis submitted to Department of Agricultural Zoology and Entomology, S.K.N. College of Agriculture, Jobner, 2010.

5. Dawae PV. Studies on biology and management of pulse beetle on stored pigeonpea M.Sc. (Ag.) thesis submitted to NDUAT, Kumarganj, Faizabad (U.P.) India, 2008.

6. Deeba F, Sarwar M, Khuhro RD. Varietal susceptibility of mungbean genotypes to pulse peetle, Callosobruchus analis (Fabricius) (Coleoptera: Bruchidae). Pak. J of Zool. 2006; 38(4):265- 268.

7. Golob P, Webley DJ. The use of plant and minerals as traditional protectants of stored products. Report of Tropical product insititute, NOG. 1980; 138:VI+32.

8. Khan MZ, Razzab M, Bhuiyan SI, Hossain MA. Eco Friendly Management of Pulse Beetle, Callosobruchus chinensis Linn. Using Botanicals on Mung bean. International Journal of Scientific and Research Publications, 2015, 5.

9. Khinchi SK, Sharma MM, Khinchi MK, Naga RP, Acharya D, Asiwal RC. Studies on efficacy of certain plant part powders against pulse beetle, Callosobruchus chinensis Linn. on chickpea, Cicer arietinum (L). Journal of Entomology and Zoology Studies. 2017; 5(3):575-578

10. Kumar R. Determination of storability index genotype against Rhizopertha domenica Fab. and its management under laboratory condition. M. Sc. (Ag.) thesis submitted to NDUAT, Kumarganj, Faizabad India, 2008.

11. Lal D, Raj DV. Efficacy of application of four vegetable oils as grain protectant against the growth and development of Callosobruchus maculatus and on its damage. Advances in Bioresearch. 2012; 3(2):55-59.

12. Pal RK, Katiyar RA. Bioefficacy of promising botanicals against pulse beetle, Callosobruchus chinensis L. infesting stored seed of moong bean. International $\mathrm{J}$ of Plant Protection. 2013; 6(2):489-491.

13. Singh RB, Nishad RN, Singh RP. Relative efficacy of botanicals against pulse beetle (Callosobruchus chinensis L.) infestation in Chickpea during Storage Bull. Env. Pharmacol. Life Sci. 2017; 6:333-336.

14. Nishad RN, Singh RB, Singh AK, Singh SP, Yadav SK. Effect of various indigenous botanical seed protectants on seed quality parameters of chickpea seed during ambient storage, Journal of Pharmacognosy and Phytochemistry. 2017; SP1:423-426.

15. Rugumamu CP. Insecticidal activity of botanical pesticides on Callosobrunchus chinensis (L.) (Coleoptera: Bruchidae) in stored green gram, Vigna radiata (L). Int. J of Agri. Sci. Res. 2015; 4(1):013-020.

16. Sarwar M, Ahmad N, Siddiqui QH, Mohammad R, Sattar $\mathrm{M}$, Tofique $\mathrm{M}$. Varietal resistance in stored mungbean against the infestation of pulse beetle, Callosobruchus analis (Fabricius) (Coleoptera: Bruchidae). Pak. J of Zool. 2003; 35(4):301- 305.

17. Shrestha UK. Need for chickpea cultivation in Nepal. In: S. Pande, C. Johansen, P. C. Stevenson and D. Grzwacz (eds.), Proceedings of the international workshop on planning and implementation of on farm IPM of chickpea in Nepal, 6-7 September, 2001, Kathmandu, Natural Resource Institute, UK and International Crops Research Institute for the Semi-Arid Tropics, India, 2001, 33-47.

18. Singh CB, Katiyar M, Pal RK, Singh SK, Singh A. Relative efficacy of neem based insecticides on seed viability and quality of pigeonpea (Cajanus cajan L.) during storage. Trends in Biosciences. 2014; 7(16):23222326.

19. Southgate BJ. The importance of the Bruchidae as pests of grain legumes, their distribution and control. In: S. R. Singh, H. F. Van Emden and T. A. Taylor (Eds.), Pests of Grain Legumes: Ecology and Control. London: Academic Press, 1978, 219-229.

20. Talekar NS. Biology, damage and control of bruchid pest of mungbean. Mungbean: Proceeding of the Second International Symposium. Asian Vegetable Research and Development Centre, Shanhua, Taiwan. AVRDC Publication No, 1988, 88-304.

21. Tesema K, Kurabachew H, Tadesse TF. Evaluation of efficacy of plant powders, cow dung, ash and malathion dust against Callosobrunchus chinensis (Coleoptera: Bruchidae) on chickpea in jole andegna: Southern Ethiopia. J of Agri. Stud. 2015; 3(2):84-88. 